\title{
DOSSIER \\ Sciences sociales et médecine : à propos des malades du sida
}

MARCEL JOLIIVET, JEAN-PAUL MOATI

$\mathrm{L}$ es deux articles qui suivent remettent en cause la division du travail entre sciences sociales et sciences médicales telle qu'elle est habituellement conçue et prônée dans toute recherche médicale interdisciplinaire et finalisée. On procède généralement en autonomisant le fait biologique (la maladie vue par le biologiste) et les procédures biotechniques que constituent le traitement (qui sont réservées aux sciences médicales), et I'on ne fait appel aux sciences sociales que pour la mise en œuvre des mesures de prévention, ce qui suppose en effet en amont une bonne connaissance des comportements sociaux en cause dans les mécanismes de la contamination et, en aval, la définition de formes d'action susceptibles d'agir sur ces comportements. Dans un tel schéma, le malade n'existe que comme organisme biologique atteint par une pathologie - et donc relevant du médecin. Or, allant à l'encontre de ce découpage, les deux articles qui suivent - qui mêlent sociologie et psychologie sociale - portent précisément sur les malades. Le premier s'intéresse directement à la façon dont les malades vivent leur maladie (une maladie particulièrement éprouvante sur le plan psychologique en raison tant de ses caractéristiques propres que du contexte biomédical dans lequel elle s'inscrit). Le second, beaucoup plus technique, traite de la façon dont les malades participent aux essais cliniques, qui représentent une des voies majeures pour la mise au point des thérapeutiques. Mais il est clair qu'à travers cette participation, c'est encore le face-à-face avec la maladie que vivent et que gèrent les malades. Ici, ce face-à-face passe clairement par un faceà-face avec la médecine - et plus concrètement - les médecins. En fait, deux attitudes se confrontent, l'une - celle des médecins qui consiste à veiller au respect rigoureux des protocoles afin de garantir la validité des résultats des essais, l'autre - celle des malades - qui consiste à utiliser les essais comme un moyen - et un moyen parmi d'autres - de vivre la maladie et de lutter contre elle, quitte à les accommoder à sa manière.

Autrement dit, c'est un malade sujet et actif que révèlent ces analyses. Et il en va de même dans le premier article qui, en fin de compte, met en évidence les formes générales de cette activité symbolique du malade et leur variabilité liée à l'histoire des individus, une histoire dont il faut souligner qu'elle est à dimensions à la fois individuelles et collectives avec, en particulier, une dimension associative forte (mais nullement exclusive). C'est donc tout un pan de la réalité de la maladie - celui que représente l'expérience du malade - que ces articles font apparaître et opposent à une vision exclusivement médicale voire éd. médicalisée 72 de celle-ci.

C'est par là même une invitation lancée aux médecins d'en tenir compte dans leurs façons de concevoir leurs rapports avec les malades et l'application des procédures thérapeutiques.

Ces considérations prennent une importance toute particulière au moment où des progrès dans la connaissance de l'évolution de la maladie (notamment en ce qui concerne la primo-infection) et dans les traitements, viennent à nouveau remettre en cause certains des repères essentiels sur lesquels les malades ont appris à fonder leurs formes de résistance psychologique à la maladie. L'allongement de la durée de vie du malade fait du sida une maladie chronique avec laquelle il faut vivre de plus en plus longtemps. L'hypothèse un moment faite d'en passer par le tirage au sort pour choisir les malades qui pourraient bénéficier du dernier traitement mis au point et consistant en l'association de trois médicaments montre à quel point le contexte social fait peser ses propres incertitudes aux côtés de celles qui résultent de la maladie elle-même sur la situation vécue par le malade.

Dans une telle démarche, les sciences sociales ne se contentent pas d'ajouter leur apport à ceux des biologistes et des médecins en se tenant en quelque sorte à l'extérieur du fait biologique et médical. Elles prennent au contraire celui-ci en charge afin de montrer comment il est aussi social et la nécessité qu'il y a de le reconnaître comme tel. Cette façon de faire a évidemment portée générale et vaut pour toute recherche interdisciplinaire. 